\title{
Inferring the microscopic surface energy of protein-protein interfaces from mutation data
}

\author{
Iain H. Moal ${ }^{1} \quad$ Justas Dapkūnas $^{2} \quad$ Juan Fernández-Recio ${ }^{1, *}$
}

${ }^{1}$ Joint BSC-IRB Research Program in Computational Biology, Life Science Department, Barcelona Supercomputing Center, C/Jordi Girona 29, 08034 Barcelona, Spain

${ }^{2}$ Institute of Biotechnology, Vilnius University, V.A. Graiciuno 8, Vilnius, LT02241, Lithuania.

Keywords: protein-protein interactions, binding affinity, interaction energy, mutation, docking, hydrophobic effect, empirical modelling

Short title: Surface energy of protein-protein interfaces

\footnotetext{
*to whom correspondence should be addressed: juanf@bsc.es
} 


\begin{abstract}
Mutations at protein-protein recognition sites alter binding strength by altering the chemical nature of the interacting surfaces. We present a simple surface energy model, parameterised with empirical $\Delta \Delta G$ values, yielding mean energies of $-48 \mathrm{cal}^{\mathrm{mol}} \mathrm{m}^{-1} . \AA^{-2}$ for interactions between hydrophobic surfaces, -51 to $-80 \mathrm{cal} . \mathrm{mol}^{-1} . \AA^{-2}$ for surfaces of complementary charge, and 66 to $83 \mathrm{cal}^{\mathrm{mol}} \mathrm{m}^{-1} . \AA^{-2}$ for electrostatically repelling surfaces, relative to the aqueous phase. This places the mean energy of hydrophobic surface burial at -24 cal.mol ${ }^{-1} . \AA^{-2}$. Despite neglecting configurational entropy and intramolecular changes, the model correlates with empirical binding free energies of a functionally diverse set of rigid-body interactions $(r=0.66)$. When used to rerank docking poses, it can place near-native solutions in the top 10 for $37 \%$ of the complexes evaluated, and $82 \%$ in the top 100 . The method shows that hydrophobic burial is the driving force for protein association, accounting for $50-95 \%$ of the cohesive energy. The model is available opensource from http://life.bsc.es/pid/web/surface_energy/and via the CCharpPPI web server http://life.bsc.es/pid/ccharppi/
\end{abstract}

\title{
1 Introduction
}

When proteins interact, part of their exteriors are transferred from an aqueous environment to the microenvironment created by each others surfaces. When the free energy of this process is positive, interfacial tension prevents aggregation at biological concentrations. When the energy is sufficiently negative, proteins can 
bind, as in recognition events evolved to perform and regulate biological functions. Many factors drive the association of proteins, and understanding them is crucial for many efforts, including but not limited to the estimation of binding affinity, $\frac{1-9}{-19}$ determining the response of biological systems to mutation, $\frac{10-19}{19}$ modulating binding selectivity, $20+33$ designing new interactions,,$\sqrt[34]{47}$ and determining the structure of macromolecular assemblies from the structures of their constituents $48-51$ Improved modelling of interaction energy would facilitate new investigation in the engineering of living systems and the study of evolution.

Since the beginning of the 19th century, it has been known that properties of macroscopic interfaces involving liquids can be explained in terms of surface tension - the cohesive forces that spans the bulk of the liquid, and the short-range forces at the interface ${ }^{53}$ By considering the forces interacting across the interface as additive, relationships between parameters of many liquid-vapour, liquidliquid, solid-liquid and even solid-solid interfaces can be deduced, such as contact angles, meniscus heights, interfacial tensions, spreading coefficients, and heats and free energies of adsorption and immersion. $[4] 60$ Interfacial tension is equivalent to the excess energy per unit area relative to the bulk, the balance of which can be determined empirically from such measurements. In the popular threecomponent model of van Oss, ${ }_{59}^{[60}$ the tension is composed of a dispersive component, and interactions between a Lewis acid component and a Lewis base component which, for instance, encapsulates the average effect of all orientations and separations of hydrogen bonds or salt bridges. The nanoscopic interfaces which characterise protein-protein interactions, adducts formed by interactions between 
neutral atoms and electron donors and acceptors (polar and charged groups), may be considered as being composed of different types of interfaces. Each interface type can be defined by different types of surfaces in contact, which contribute additively to the overall binding affinity in proportion to their area. While each complex differs in atomic detail, the interface types are common between complexes, allowing the inference of interface energies from experimental data via a system of linear equations.

Outline: In this work, we present a simple surface energy model based on a microscopic interpretations of the van Oss model. The average per square Ångstrom free energy contribution of each interface type is calculated from empirical mutation data, and corresponds to the energy of cohesion of the two surface types minus their energy of solvation. Protein surfaces are partitioned according to four atom types, corresponding to the terms in the approach of van Oss (H-bond donors, acceptors, donor and acceptors, and neutral). In turn, this allows the interface to be partitioned according to the ten types of interaction that can occur between the four surface types. Energies for each interaction type are inferred by comparing changes in binding free energy upon mutation to changes in interaction type areas upon mutation using an approach recently developed for inferring contact energies .61 The values are compared with estimates of the energy associated with the burial of hydrophobic surface area from simulation, 62 oil/water transfer, 63 -67 and small mutational studies,,$\frac{68,-70}{70}$ as well as binding efficiency values derived from protein affinity datasets $\frac{71,72}{72}$ and alanine-scanning experiments. $\frac{73,74}{7}$ Further, we 
use the approach to estimate the contribution made by interface burial to proteins of known binding affinity, as well as evaluate the ability of the model to rerank docked poses derived from unbound protein-protein docking.

\section{Material and Methods}

\subsection{Overview}

With crystal structures of protein complexes for which experimental mutation data is available ${ }^{75}$ the protein-protein interface is calculated using Voronoï decomposition ${ }^{76,77}$ and the total surface area of each surface type is determined. The mutant form is modelled and the surface type composition of the mutant is calculated. The wild-type and mutant surfaces are used to define an expression relating the change in surface type compositions to $\Delta \Delta G$, the change in binding free energy upon mutation, where each surface types contribution to binding energy is proportional to its area. Such as equation can be constructed for each mutant in our training set, and interfacial tensions are found that maximise the agreement between experimental and theoretical energies. The process is depicted in Figure 1 with further details given below.

\subsection{Data set of changes in binding affinity upon mutation}

Mutation data were taken from the SKEMPI database, ${ }^{75}$ in which 3047 experimental $\Delta \Delta G$ values are available, in addition to their corresponding wild-type 
crystal structures. Values corresponding to unusual experimental techniques were removed (values for the jel42-FAB/HPr complex, 2JEL, and apparent affinities for the factor VIIa/soluble tissue factor complex, 1DAN). This data set was filtered so as to only include mutants with mutations at the interface region; we classified mutated residues according to their location using the method of Levy ${ }^{78}$ and removed data for mutants containing a mutation in the protein interior or support region of the interface, as well as mutants with more than three mutations at the rim or core of the interface. We also removed mutants with mutations to or from proline, due to the structural changes and energetic effects associated with $\phi$ and $\psi$ angle restriction. This left us with 1949 mutations as described previously! 61 To model the mutant protein from the wild-type structure, side-chains were re-

placed and optimised using FoldX ${ }^{79}$ Finally, we additionally filtered to remove mutants with clashes upon mutation $\left(>4.0 \mathrm{kcal}^{\mathrm{mol}}{ }^{-1}\right.$ in the FoldX van der Waals repulsion term), as we aim only to model the dispersion component of the van der Waals energy, leaving $1890 \Delta \Delta G$ values for training the model. These data are shown in Table $\mathrm{S} 1$.

\subsection{Interface type calculation}

Atoms were classified by an atom typing scheme similar to that used by Jiang et al, $\frac{80}{}$ as either hydrogen bond acceptors (carbonyl oxygens in the peptide backbone and asparagine and glutamine side-chains, carboxyl oxygens in the side-chains of aspartic acid and glutamic acid, and the $\delta$ nitrogen of histidine), hydrogen bond donors (amide nitrogens in the peptide backbone, the indole nitrogen in trypto- 
phan, amine nitrogens in asparagine, glutamine and lysine, the $\varepsilon$ nitrogen of histidine and the guanidinium nitrogens of arginine), hydrogen bond donor and acceptors (alcohol oxygens of tyrosine, threonine and serine) and neutral (all remaining atoms). Interactions between the four atom types give rise to ten interaction types. Intermolecular atomic contact areas for each interaction type were determined using Voronoï diagrams, calculated using the CAD-score program.76] 77 Interface areas for each interaction type are given in Table S1. The total intermolecular contact area correlated with changes in solvent accessible surface area upon binding taken from the DockGround server, $\frac{81}{2}$ with slope and intercept close to unity and zero respectively for a large set of complexes (Figure S1).

\subsection{Surface energy calculation}

The presented method is a model of the binding free energy components which arise from the interacting surface, modelled as the sum of contributions from each interaction type, which are each linearly proportional to their interaction area.

$$
\Delta G_{\text {surf }}=\sum_{i=1}^{4} \sum_{j=1}^{i} \gamma_{i, j} A_{i, j}
$$

where the summation runs over atom types, $A_{i, j}$ is the interaction area between atoms of type $i$ and $j$, and the $\gamma_{i, j}$ constant is the microscopic interfacial tension in units of kcal.mol ${ }^{-1} . \AA^{-2}$. We calculate the interfacial tensions by relating the change in binding free energy upon mutation, $\Delta \Delta G$, to the changes in interacting

surface areas. For instance, if a mutation with $\Delta \Delta G=1.5 \mathrm{kcal}^{\mathrm{m}} \mathrm{mol}^{-1}$ corresponds 
to the loss of $10 \AA^{2}$ of surface area between atom type $i$ and atom type $j$, and the creation of $5 \AA^{2}$ of surface area between atom type $i$ and atom type $k$, we can say that $5 \gamma_{i, k}-10 \gamma_{i, j}=1.5+r$, where $r$ is the residual error. Such an equation can be defined for each mutant in the training set, as

$$
\Delta \Delta G_{\text {surf }}=r+\sum_{i=1}^{4} \sum_{j=1}^{i} \gamma_{i, j}\left(A_{i, j}^{\text {mutant }}-A_{i, j}^{\text {wild-type }}\right)
$$

Using this series of equations, we calculate $\hat{\gamma}$, the vector of $\gamma$ values which minimise the sum square of the residual errors, $\sum r^{2}$, thus yielding the interfacial tension values which give the greatest agreement with the experimental binding free energy changes upon mutation, given model assumptions. This is achieved using least squares as described in Moal and Fernández-Recio 61

$$
\hat{\gamma}=\left(\mathbf{A}^{\mathrm{T}} \mathbf{A}\right)^{-1} \mathbf{A}^{\mathrm{T}} \mathbf{E}
$$

where $\mathbf{A}$ is a matrix of changes in interaction type areas upon mutation and $\mathbf{E}$ is a vector of $\Delta \Delta G$ values. Unlike in our previous study, a weight matrix was not included as it did not improve the model. This is likely due to the much smaller number of parameters being fit, resulting in a model that is less susceptible to fitting towards overrepresented residues, complexes and families. To improve the stability of the training and derive model parameter deviations, bootstrap aggregating was performed, 1000 bootstrap samples of the mutation were taken and used to obtain model parameters via equation 3 , from which final $\hat{\gamma}$ values were found by averaging. 
In terms of physical interpretation, the calculated surface energy is dominated by effects relating to interface desolvation and the enthalpy arising from the physical contact of the binding partners. We justify this interpretation by considering the other sources of energy in the context of the model; all the mutations used correspond to those for which binding is observed for both the wild-type and mutant protein, and thus the rotational, translational and backbone restrictions are similar in both, and their entropic contributions approximately cancel. The same holds for side-chain restriction of residues away from the mutated site. Regarding the change in binding configurational entropy upon mutation for the the sidechain being mutated, estimates of the entropy of conformationally unrestricted side-chains $\frac{83}{86}$ are around $0.9 \mathrm{kcal}^{\mathrm{mol}} \mathrm{mol}^{-1}$. In the training data, some of this contribution is cancelled when subtracting the wild-type energy from the mutant. In addition, side-chains are already somewhat conformationally restricted in the folded unbound state, and residual flexibility still exists in the bound, thus the difference in side-chain entropy change of the mutated residue is likely to be small. Given that the model is unlikely to be able to infer this component using changes in surface area types, it is unlikely to contribute significantly to the calculated surface energies.

\subsection{Absolute binding affinity benchmark}

Experimental affinity data for a total of 240 complexes was taken from two data

sources. The binding affinity benchmark reported in Kastritis et al ${ }^{87}$ has data for 144 non-redundant high resolution complexes, for which unbound structures 
are also available. Six complexes were removed: 2OZA, due to large disorderto-order transitions upon binding, 2PTC and 2SNI, protease inhibitors whose anomalously high affinity likely relate to interactions with the catalytic machinery, 1BRS, due to the high electrostatic association contribution of residues not

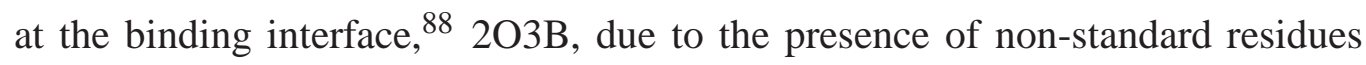
and an ion at the binding interface, and 1EER, due to its poor wwPDB X-ray Structure Validation Report including a large number of Ramachandran outliers, clashes and non-rotameric side-chains. The affinity benchmark is subclassified by conformational similarity to the unbound structures, as flexible (interface RMSD $>1.0 \AA \AA$ ) or rigid (interface RMSD $<1.0 \AA \AA$ ), as reported in Kastritis et al. ${ }^{87}$ The benchmark is also subclassified as validated or non-validated, depending on the confidence with which the experimental values are know, with validated affinities being those for which similar values have been reported in the literature by at least two research groups or experimental techniques, and can thus be thought of as a consensus in the literature, as described in Moal et al. ${ }^{[3}$ The second set of binding affinity data used is a set of 113 complexes reported in Chen et al. ${ }^{71}$ Complexes already in the affinity benchmark were removed, as were four proteinpeptide complexes involving non-standard amino acids (2R0Y, 1QNG, 2WFJ and 3JVK), leaving 102 complexes. These were further subdivided by interface size into 72 small $\left(<1800 \AA^{2}\right)$ and 30 large $\left(>1800 \AA^{2}\right)$ complexes. Only two of the complexes in the Chen set had high homology with those in the affinity benchmark ( $>45 \%$ sequence identity and $>5$ matched amino acids). One of these, 10P9, is a lysozyme/antibody complex similar to 1VFB. Examination of the structure re- 
vealed that the two antibodies target different epitopes. The other is the colicin E9 / IM9 interaction 2VLQ, which is similar to 1EMV and the non-cognate E9 / IM2 interaction, 2WPT, which is six orders of magnitude lower in affinity. Removing 2VLQ from the data set resulted in an insignificant improvement in the correlation ( $\mathrm{r}$ increases by 0.003 ), and thus has no effect on the conclusions reached.

In applying the surface energy model to absolute binding affinity data, equation 1 was used. All complexes, affinities and interface type areas are given in Table S2.

\subsection{Docking decoys}

The docking decoys used for reranking were previously generated using the flexible guided docking algorithm SwarmDock ${ }^{89}[91$ using the standard protocol, applied to 176 unbound structures in the protein-protein docking benchmark 4.0.92 Decoys were classified as high quality, medium quality, acceptable or incorrect according to the CAPRI criteria. ${ }^{93}$ Complexes for which acceptable or better solutions could not be found were not considered, resulting in decoy sets for 123 complexes, of around 500 poses each. Each pose was scored and ranked using equation 1 , and clustered as per the standard procedure. ${ }^{89}$ 


\section{Results and Discussion}

\subsection{Surface free energies}

After fitting the model, the Pearson correlation with the $\Delta \Delta G$ data is 0.40 (Figure S2). While low, this value compares reasonably with similar studies. For instance, using the same source data set and removing outliers in the P1 position of the trypsin-BPTI complex, one study achieved correlations of 0.47 to $0.55 ! \frac{18}{1}$ In another study predicting the effects of mutation on folding free energy, Dehouck et al compared their methods to five others with similarly modest correlations of 0.27 to 0.48 .94 Nevertheless, most the derived surface energy values, which are displayed and tabulated in Figure 2, are intuitively plausible given the error estimates. For instance, favourable interactions are observed between opposing charges (-51, -65 and -79 cal.mol $^{1} . \AA^{-2}$ for acceptor/donor, donor/hydroxyl and acceptor/hydroxyl interactions respectively) and between hydrophobic surfaces (-

48 cal.mol $\left.{ }^{1} . \AA^{-2}\right)$, and unfavourable energies between like charges (+64 and +83 cal.mol ${ }^{1} . \AA^{-2}$ for donor/donor and acceptor/acceptor interactions respectively), all of which are true even at the extremes of the $95 \%$ confidence interval $(1.96 \sigma)$. The total sum of absolute change in interactions area across the data set amounts to $3152 \mathrm{~nm}^{2}$. However, this is not equally distributed across interaction types. For instance, $42 \%$ of this corresponds to the creation or removal of interactions between two hydrophobic surfaces, with a further $41 \%$ corresponding to polarneutral interactions, and $10 \%$ corresponding to acceptor-donor surface. Thus, the corresponding error estimates are lowest for these types of interaction. On the 
other hand, the relative rarity of hydroxyl groups means that their interactions are less represented in the data set and less confidence can be ascribed to their energies. For instance, there is only $6 \mathrm{~nm}^{2}$ of change in hydroxyl-hydroxyl surface in the training set, and the surface energy for this interaction type at the $95 \%$ confidence interval could be anywhere between -97 and +99 cal.mol ${ }^{-1} . \AA^{-2}$, revealing virtually nothing about this interaction. Further, accounting for the errors in the low values for the hydroxyl-acceptor and hydroxyl-donor interactions the energy could reasonably be as high as the $-40 \mathrm{cal}^{\mathrm{mol}} \mathrm{l}^{-1} . \AA^{-2}$ mark, a range which eclipses the more precise energy calculated for the acceptor-donor interaction, -50 cal.mol ${ }^{-1} . \AA^{-2}$, and the estimate of Guharoy and Chakrabarti 73 at -52 cal.mol ${ }^{-1} . \AA^{-2}$.

While the interaction types discussed above are in accordance with chemical intuition, it would seem hard, at first glance, to reconcile the seemingly low energies of neutral-polar interactions, considering the rarity of unpaired hydrogen bond donors and acceptors at protein cores; the energies for the neutral-acceptor and neutral-donor interaction types are small, and the neutral-hydroxyl interaction appears stabilising, even after accounting for errors. However neutral-polar contacts are common in protein-protein interactions, whose interfaces are intermediate in composition between the protein core and non-interacting surface .95 There are a number of considerations which should be made. Firstly, one major difference between binding free energies and the microscopic surface free energy model derived here is that the latter ignores destabilising entropy effects. Translational and rotational degrees of freedom are also lost and only partially recovered 
as internal modes. Further entropic penalties arise from restricting the number of accessible rotameric states and fluctuations within accessible rotamers, as well as global modes. Assuming that the magnitude of these effects is approximately equivalent in the wild-type and mutant structures in the training data, the nearzero energies reported here are negligible considering the whole binding process. Secondly, evolutionary pressures to maintain hydrogen bonding seem weaker for protein-protein interactions than for folds, as structural interologs comparisons show that intermolecular hydrogen bonds are not highly conserved. ${ }^{99}$ Further, interface rim residues, which constitute half of the data set, are partially solvent exposed, unlike protein interiors. Even the core interface residues themselves are frequently in contact with buried waters, which are commonly trapped at interfaces. .100

One aspect of this work pertains to the magnitude of the hydrophobic factor in stabilising proteins and their assemblies, postulated by Langmuir 101 brought to the fore by Kauzmann. ${ }^{102}$ How this manifests in protein-protein interactions, where hydrophobic patches in protein-protein interactions vary greatly in

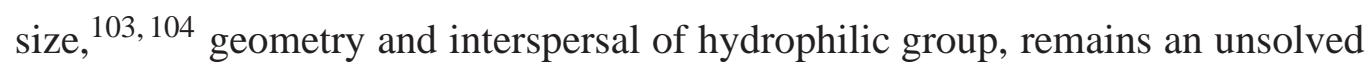
problem $\cdot{ }^{105}$ Nevertheless the magnitude of the hydrophobic effect, as determined by amino acid oil/water transfer experiments, is proportional to the area of buried hydrophobic surface, with initial estimates of $24 \mathrm{cal}^{\mathrm{m}} \mathrm{mol}^{-1} . \AA^{-2}[\underline{63}$ However, different solvents give different transfer energies and it is uncertain whether the interior of a binding interface can be modelled as an organic liquid, or how best to relate transfer energies to protein desolvation energies ${ }^{106}$ Thus, estimates vary 


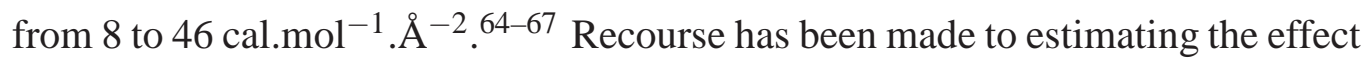
through molecular simulation, yielding a similar estimate of $24 \mathrm{cal}_{\mathrm{mol}} \mathrm{mol}^{-1} . \AA^{-2}, \underline{62}$ and through mutation experiments. These latter investigations have only been undertaken on a small number of mutations in specific systems, again yielding a

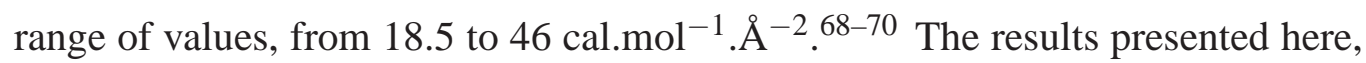
averaged over 1890 mutation, indicate a mean hydrophobic energy of $24+/-2.4$ cal.mol ${ }^{-1} . \AA^{-2}$, lending support to the median values derived from transfer, simulation and single mutation approaches.

\subsection{Application to absolute binding affinities}

The model was applied to the complexes with experimentally determined affinity, with results shown in Figure 3 and correlations summarised in Table 1 As the model does not account for intramolecular energy changes, the correlations with the rigid complexes are higher than with the flexible, as expected. Further, the correlations with the validated data is higher than for the complexes for which affinities were reported only once, or for which conflicting affinities exist within the literature. Indeed, the only category for which the correlation is not significant is the intersection between the unvalidated set and the set of flexible complexes. A number of recent empirical affinity models have been trained directly from affinity data, achieving correlations in the range of $0.45-0.65$, and up to around 0.7 when considering only rigid-body interactions. 2, 3,6]107 Although these binding energy models are not directly comparable to the surface energy model presented here, they give an indication of the abilities of current empirical functions. 
One salient observation is that the proposed model generates energies that are much lower than observed. This of itself comes as no surprise, as the model does not account for changes in rotational, translational and conformational entropy changes. However, observing the least-squares trend line in Figure 3A, this discrepancy arises from a shallow slope. If the regression could be reliably extrapolating to the intercept, this would lead to the absurd conclusion that even without any interacting surface area, the proteins would have a binding affinity of -5 kcal.mol ${ }^{-1}$. This is surprising, as the loss of external (rotational and translational) entropy depends only on the restriction in protein orientation and position relative to the binding partner, which should be roughly constant and not related to size of the binding surface; the cost of this is on the order of 10 to $15 \mathrm{kcal}^{\mathrm{mol}}{ }^{-1}$, under the reasonable assumption that unbound proteins are translationally and rotationally unrestricted and amenable to the Sackur-Tetrode equation, 108 even accounting for residual freedom, 109 although some experimental data suggests lower penalties. This physical implausibility is also observed applying linear least square regression to all the parameters which correlate with binding affinity in a previous study, $\frac{3}{3}$ as well as the empirically trained models of Audie and Scarlata ${ }^{7}$ and, despite indications in the text suggesting otherwise, Horton and Lewis. ${ }^{8}$ Further investigation into this apparent missing energy is required.

Conformational entropy is harder to calculate or derive a ballpark estimate for. Generally, this also decreases upon binding due to restrictions of global backbone motions, the number of accessible rotameric states and within-rotamer motions $110-112$ Although more variable, a difference is to be expected between the 
flexible and rigid categories of interactions. The rigid category is composed of proteins which either have limited flexibility or are flexible and have observed apo structures that are, by chance, crystalographically fixed into a conformation near their holo structure. The flexible category, on the other hand, only contains proteins that are inherently flexible. Thus, in the latter, we would expect a greater disparity in magnitude between the calculated surface energies and observed binding free energies, which is indeed the case (Figure $3 \mathrm{~A}$ ).

\subsection{The Hydrophobic effect drives binding}

The model offers a method of partitioning the attractive forces by surface type. In order to investigate the driving forces in protein adhesion, it was applied to 4434 interactions downloaded from the DockGround server. ${ }^{81}$ A sequence identity cutoff of $30 \%$ was used to remove redundancies on each chain, and complexes with less that $2.5 \AA$ resolution or interface areas outside of the 500-6000 $\AA^{2}$ were also filtered. The relative contributions from the various components are shown in Figure 4. Energy from hydrophobic contacts was by far the greatest contributor, constituting $50 \%$ to $95 \%$ of the negative energy. This is in agreement with earlier

studies where a smaller number of systems were investigated. 113, 114

The computed surface energies correlate strongly with buried surface area $(\mathrm{r}=0.9)$, which in turn also correlates with binding affinity (Table 1). The differences between the two metrics yield slightly greater correlation for the model compared to the buried area, although this is not statistically significant $(\mathrm{p}=0.13$, $\mathrm{t}=-1.14$, significance of difference between paired correlations test, $\mathrm{r}_{x y}=0.43, \mathrm{r}_{x z}=0.4$, 
$\left.\mathrm{r}_{y z}=0.9, \mathrm{n}=240\right)$.

\subsection{Re-ranking docked poses}

Proteins are able to locate and form long lasting complexes with their specific binding partners while not binding to the many other proteins which they encounter and make transient interactions with. Thus, a critical test of a binding model is its ability to distinguish specific binding modes from non-specific modes. Although the correlations of the presented model with the absolute binding affinity data reported above are only marginally higher than those for buried surface area, the latter fails this test. Distinguishing between specific and non-specific binding is closely related to the problem of ranking of docked poses, to which the model was applied. Results are shown in Table 2. Top 1/10/100 acceptable or better success rates correspond to $11 \%, 37 \%$ and $82 \%$ of the 123 complexes for which such a solution could be found by SwarmDock. Performing the same clustering and ranking procedure using only buried surface area gave success rates of $4 \%, 25 \%$ and $67 \%$. Our success rate corresponds to $8 \%, 26 \%$ and $57 \%$ of the whole benchmark of 176 complexes. ${ }^{[2]}$ For comparison, the highest performing

model reported in Moal and Fernández-Recio ${ }^{61}$ had success rates of $6 \%, 11 \%$ and $56 \%$ using the same decoy set, and the reported success rates for decoys generated using the PatchDock-FireDock protocol on the same benchmark were 10\%, 24\% and $49 \% .115$ Thus, the model performs comparably to a popular docking protocol despite not being optimised for this task.

To investigate whether the docking performance was influenced by training 
the potential using mutations for the complexes being docked, we recalculated the potential and reranked the decoys for the 32 complexes for which such mutations existed in the training set. In these cases, mutations in the complex being docked were omitted from the training, as were those in homologous proteins $(>45 \%$ sequence identity in both chains). The training sets for the recalculated potentials contained at least 1706 mutations, and only small changes in the surface energies were observed. The top 10 acceptable or better success rate was the same as when the potential was trained with all mutants, indicating that having these data in the training set does not influence docking success, and that the model is robust to small perturbations in the training set.

\section{Conclusion}

In summary, we have presented a simple interacting surface energy model based on interactions between four different surface patches (neutral, donor, acceptor and donor/acceptor), in which the energy of interacting surfaces is proportional to the interacting surface area. Constants of proportionality are derived using mutations for which experimental changes in binding free energies are available, and changes in interaction areas are modelled by in silico mutagenesis from wild-type crystal structures. The model yields realistic parameters for electrostatic repelling and attracting surfaces, and hydrophobic burial energy in quantitative agreement with value derived from oil/water transfer, simulation and small mutational studies. When applied across whole binding interfaces, the model yields values which 
correlate with absolute binding free energies, as the interacting surface makes a major contribution to the overall energy of binding. The model gives slightly stronger correlations than total buried surface area, although it distinguishes itself from the measure by being able to rank docked poses, thereby discriminating between specific and non-specific binding modes. Applied to a larger data set, the method indicates that the hydrophobic force is the major driver of protein binding, in accord with previous studies. ${ }^{113] 114}$ The model is accessible via the CCharPPI web server ${ }^{116}$ and an open source implementation can be downloaded to be run locally from http://life.bsc.es/pid/web/surface_energy/

\section{Acknowledgements}

This research was in part supported by postdoctoral fellowship funded by European Union Structural Funds project "Postdoctoral Fellowship Implementation in Lithuania". The research leading to these results has received funding from the People Programme (Marie Curie Actions) of the European Unions Seventh Framework Programme (FP7/2007-2013) under REA grant agreement PIEF-GA2012-327899, and the Spanish Ministry of Science and Innovation project [BIO201348213-R]. The authors declare no conflict of interests. 


\section{References}

[1] Lensink MF, Wodak SJ. Docking, scoring, and affinity prediction in CAPRI. Proteins 2013;81:2082-2095.

[2] Vreven T, Hwang H, Pierce BG, Weng Z. Prediction of protein-protein binding free energies. Protein Sci 2012;21:396-404.

[3] Moal IH, Agius R, Bates PA. Protein-protein binding affinity prediction on a diverse set of structures. Bioinformatics 2011;27:3002-3009.

[4] Moal IH, Bates PA. Kinetic rate constant prediction supports the conformational selection mechanism of protein binding. PLoS Comput Biol 2012; 8:e1002351.

[5] Audie J, Swanson J. Advances in the prediction of protein-peptide binding affinities: implications for peptide-based drug discovery. Chem Biol Drug Des 2013;81:50-60.

[6] Kastritis PL, Rodrigues JP, Folkers GE, Boelens R, Bonvin AM. Proteins feel more than they see: fine-tuning of binding affinity by properties of the non-interacting surface. J Mol Biol 2014;426:2632-2652.

[7] Audie J, Scarlata S. A novel empirical free energy function that explains and predicts protein-protein binding affinities. Biophys Chem 2007; 129:198-211. 
[8] Horton N, Lewis M. Calculation of the free energy of association for protein complexes. Protein Sci 1992;1:169-181.

[9] May A, Pool R, van Dijk E, Bijlard J, et al. Coarse-grained versus atomistic simulations: realistic interaction free energies for real proteins. Bioinformatics 2014;30:326-334.

[10] Sharabi O, Shirian J, Shifman JM. Predicting affinity- and specificityenhancing mutations at protein-protein interfaces. Biochem Soc Trans 2013;41:1166-1169.

[11] Sharabi O, Peleg Y, Mashiach E, Vardy E, et al. Design, expression and characterization of mutants of fasciculin optimized for interaction with its target, acetylcholinesterase. Protein Eng Des Sel 2009;22:641-648.

[12] Sharabi O, Dekel A, Shifman JM. Triathlon for energy functions: who is the winner for design of protein-protein interactions? Proteins 2011; 79:1487-1498.

[13] Qin S, Zhou HX. Using the concept of transient complex for affinity predictions in CAPRI rounds 20-27 and beyond. Proteins 2013;81:2229-2236.

[14] Moretti R, Fleishman SJ, Agius R, Torchala M, et al. Community-wide evaluation of methods for predicting the effect of mutations on proteinprotein interactions. Proteins 2013;81:1980-1987.

[15] Pallara C, Jimenez-Garcia B, Perez-Cano L, Romero-Durana M, et al. Expanding the frontiers of protein-protein modeling: from docking and scor- 
ing to binding affinity predictions and other challenges. Proteins 2013; 81:2192-2200.

[16] Kilambi KP, Pacella MS, Xu J, Labonte JW, et al. Extending RosettaDock with water, sugar, and $\mathrm{pH}$ for prediction of complex structures and affinities for CAPRI rounds 20-27. Proteins 2013;81:2201-2209.

[17] Agius R, Torchala M, Moal IH, Fernandez-Recio J, Bates PA. Characterizing changes in the rate of protein-protein dissociation upon interface mutation using hotspot energy and organization. PLoS Comput Biol 2013; 9:e1003216.

[18] Dehouck Y, Kwasigroch JM, Rooman M, Gilis D. BeAtMuSiC: Prediction of changes in protein-protein binding affinity on mutations. Nucleic Acids Res 2013;41:W333-339.

[19] Pires DE, Ascher DB, Blundell TL. mCSM: predicting the effects of mutations in proteins using graph-based signatures. Bioinformatics 2014; $30: 335-342$.

[20] Sharabi O, Erijman A, Shifman JM. Computational methods for controlling binding specificity. Meth Enzymol 2013;523:41-59.

[21] Yosef E, Politi R, Choi MH, Shifman JM. Computational design of calmodulin mutants with up to 900-fold increase in binding specificity. J Mol Biol 2009;385:1470-1480. 
[22] Fromer M, Shifman JM. Tradeoff between stability and multispecificity in the design of promiscuous proteins. PLoS Comput Biol 2009;5:e1000627.

[23] Procko E, Hedman R, Hamilton K, Seetharaman J, et al. Computational design of a protein-based enzyme inhibitor. J Mol Biol 2013;425:35633575 .

[24] Fridman Y, Gur E, Fleishman SJ, Aharoni A. Computational protein design suggests that human PCNA-partner interactions are not optimized for affinity. Proteins 2013;81:341-348.

[25] Potapov V, Reichmann D, Abramovich R, Filchtinski D, et al. Computational redesign of a protein-protein interface for high affinity and binding specificity using modular architecture and naturally occurring template fragments. J Mol Biol 2008;384:109-119.

[26] Mandell DJ, Kortemme T. Computer-aided design of functional protein interactions. Nat Chem Biol 2009;5:797-807.

[27] Sammond DW, Eletr ZM, Purbeck C, Kuhlman B. Computational design of second-site suppressor mutations at protein-protein interfaces. Proteins 2010;78:1055-1065.

[28] Havranek JJ, Harbury PB. Automated design of specificity in molecular recognition. Nat Struct Biol 2003;10:45-52.

[29] Grigoryan G, Reinke AW, Keating AE. Design of protein-interaction specificity gives selective bZIP-binding peptides. Nature 2009;458:859-864. 
[30] Kiel C, Filchtinski D, Spoerner M, Schreiber G, et al. Improved binding of raf to Ras.GDP is correlated with biological activity. J Biol Chem 2009; 284:31893-31902.

[31] Tharakaraman K, Robinson LN, Hatas A, Chen YL, et al. Redesign of a cross-reactive antibody to dengue virus with broad-spectrum activity and increased in vivo potency. Proc Natl Acad Sci USA 2013;110:E1555-1564.

[32] Lewis SM, Wu X, Pustilnik A, Sereno A, et al. Generation of bispecific $\mathrm{IgG}$ antibodies by structure-based design of an orthogonal Fab interface. Nat Biotechnol 2014;32:191-198.

[33] Karanicolas J, Kuhlman B. Computational design of affinity and specificity at protein-protein interfaces. Curr Opin Struct Biol 2009;19:458-463.

[34] Strauch EM, Fleishman SJ, Baker D. Computational design of a pHsensitive IgG binding protein. Proc Natl Acad Sci USA 2014;111:675-680.

[35] Schreiber G, Fleishman SJ. Computational design of protein-protein interactions. Curr Opin Struct Biol 2013;23:903-910.

[36] Der BS, Kuhlman B. Strategies to control the binding mode of de novo designed protein interactions. Curr Opin Struct Biol 2013;23:639-646.

[37] Whitehead TA, Baker D, Fleishman SJ. Computational design of novel protein binders and experimental affinity maturation. Meth Enzymol 2013; 523:1-19. 
[38] Khare SD, Fleishman SJ. Emerging themes in the computational design of novel enzymes and protein-protein interfaces. FEBS Lett 2013;587:11471154.

[39] Fleishman SJ, Whitehead TA, Strauch EM, Corn JE, et al. Communitywide assessment of protein-interface modeling suggests improvements to design methodology. J Mol Biol 2011;414:289-302.

[40] Fleishman SJ, Whitehead TA, Ekiert DC, Dreyfus C, et al. Computational design of proteins targeting the conserved stem region of influenza hemagglutinin. Science 2011;332:816-821.

[41] Fleishman SJ, Corn JE, Strauch EM, Whitehead TA, et al. Hotspot-centric de novo design of protein binders. J Mol Biol 2011;413:1047-1062.

[42] Jacobs TM, Kuhlman B. Using anchoring motifs for the computational design of protein-protein interactions. Biochem Soc Trans 2013;41:11411145 .

[43] London N, Ambroggio X. An accurate binding interaction model in de novo computational protein design of interactions: if you build it, they will bind. J Struct Biol 2014;185:136-146.

[44] Karanicolas J, Corn JE, Chen I, Joachimiak LA, et al. A de novo protein binding pair by computational design and directed evolution. Mol Cell 2011;42:250-260. 
[45] Stranges PB, Machius M, Miley MJ, Tripathy A, Kuhlman B. Computational design of a symmetric homodimer using beta-strand assembly. Proc Natl Acad Sci USA 2011;108:20562-20567.

[46] Guntas G, Purbeck C, Kuhlman B. Engineering a protein-protein interface using a computationally designed library. Proc Natl Acad Sci USA 2010; 107:19296-19301.

[47] Jardine J, Julien JP, Menis S, Ota T, et al. Rational HIV immunogen design to target specific germline B cell receptors. Science 2013;340:711-716.

[48] Ritchie DW. Recent progress and future directions in protein-protein docking. Curr Protein Pept Sci 2008;9:1-15.

[49] Halperin I, Ma B, Wolfson H, Nussinov R. Principles of docking: An overview of search algorithms and a guide to scoring functions. Proteins 2002;47:409-443.

[50] Moal IH, Torchala M, Bates PA, Fernandez-Recio J. The scoring of poses in protein-protein docking: current capabilities and future directions. BMC Bioinformatics 2013;14:286.

[51] Flores SC, Bernauer J, Shin S, Zhou R, Huang X. Multiscale modeling of macromolecular biosystems. Brief Bioinformatics 2012;13:395-405.

[52] Moal IH, Moretti R, Baker D, Fernandez-Recio J. Scoring functions for protein-protein interactions. Curr Opin Struct Biol 2013;23:862-867. 
[53] Young T. An Essay on the Cohesion of Fluids. Philos Trans R Soc Lond 1805;95:65-87.

[54] Owens DK, Wendt RC. Estimation of the surface free energy of polymers. J Appl Polym Sci 1969;13:1741-1747.

[55] Fowkes FM. Attractive forces at interfaces. Ind Eng Chem Res 1964; $56: 40-52$.

[56] Fowkes FM. Donor-acceptor interactions at interfaces. J Adhesion 1972; $4: 155-159$.

[57] Wu S. Calculation of interfacial tension in polymer systems. J Polym Sci, Part C: Polym Sym 1971;34:19-30.

[58] Wu S. Polar and nonpolar interactions in adhesion. J Adhesion 1973;5:3955.

[59] Van Oss CJ, Chaudhury MK, Good RJ. Interfacial lifshitz-van der waals and polar interactions in macroscopic systems. Chem Rev 1988;88:927941.

[60] Oss CV, Good R, Chaudhury M. The role of van der waals forces and hydrogen bonds in between biopolymers and low energy surfaces. J Colloid Interface Sci 1986;111:378 - 390. 
[61] Moal IH, Fernandez-Recio J. Intermolecular contact potentials for proteinprotein interactions extracted from binding free energy changes upon mutation. J Chem Theory Comput 2013;9:3715-3727.

[62] Raschke TM, Tsai J, Levitt M. Quantification of the hydrophobic interaction by simulations of the aggregation of small hydrophobic solutes in water. Proc Natl Acad Sci USA 2001;98:5965-5969.

[63] Chothia C. Hydrophobic bonding and accessible surface area in proteins. Nature 1974;248:338-339.

[64] Hermann RB. Use of solvent cavity area and number of packed solvent molecules around a solute in regard to hydrocarbon solubilities and hydrophobic interactions. Proc Natl Acad Sci USA 1977;74:4144-4145.

[65] Eisenberg D, McLachlan AD. Solvation energy in protein folding and binding. Nature 1986;319:199-203.

[66] Sharp KA, Nicholls A, Fine RF, Honig B. Reconciling the magnitude of the microscopic and macroscopic hydrophobic effects. Science 1991;252:106109.

[67] Ooi T, Oobatake M, Nemethy G, Scheraga HA. Accessible surface areas as a measure of the thermodynamic parameters of hydration of peptides. Proc Natl Acad Sci USA 1987;84:3086-3090.

[68] Chakravarty S, Bhinge A, Varadarajan R. A procedure for detection and quantitation of cavity volumes proteins. Application to measure the 
strength of the hydrophobic driving force in protein folding. J Biol Chem 2002;277:31345-31353.

[69] Sundberg EJ, Urrutia M, Braden BC, Isern J, et al. Estimation of the hydrophobic effect in an antigen-antibody protein-protein interface. Biochemistry 2000;39:15375-15387.

[70] Li Y, Huang Y, Swaminathan CP, Smith-Gill SJ, Mariuzza RA. Magnitude of the hydrophobic effect at central versus peripheral sites in proteinprotein interfaces. Structure 2005;13:297-307.

[71] Chen J, Sawyer N, Regan L. Protein-protein interactions: general trends in the relationship between binding affinity and interfacial buried surface area. Protein Sci 2013;22:510-515.

[72] Day ES, Cote SM, Whitty A. Binding efficiency of protein-protein complexes. Biochemistry 2012;51:9124-9136.

[73] Guharoy M, Chakrabarti P. Conservation and relative importance of residues across protein-protein interfaces. Proc Natl Acad Sci USA 2005; 102:15447-15452.

[74] Guharoy M, Chakrabarti P. Empirical estimation of the energetic contribution of individual interface residues in structures of protein-protein complexes. J Comput Aided Mol Des 2009;23:645-654. 
[75] Moal IH, Fernandez-Recio J. SKEMPI: a Structural Kinetic and Energetic database of Mutant Protein Interactions and its use in empirical models. Bioinformatics 2012;28:2600-2607.

[76] Olechnovic K, Kulberkyte E, Venclovas C. CAD-score: a new contact area difference-based function for evaluation of protein structural models. Proteins 2013;81:149-162.

[77] Olechnovic K, Venclovas C. Voronota: A fast and reliable tool for computing the vertices of the Voronoi diagram of atomic balls. J Comput Chem 2014;35:672-681.

[78] Levy ED. A simple definition of structural regions in proteins and its use in analyzing interface evolution. J Mol Biol 2010;403:660-670.

[79] Guerois R, Nielsen JE, Serrano L. Predicting changes in the stability of proteins and protein complexes: a study of more than 1000 mutations. J Mol Biol 2002;320:369-387.

[80] Jiang L, Gao Y, Mao F, Liu Z, Lai L. Potential of mean force for proteinprotein interaction studies. Proteins 2002;46:190-196.

[81] Douguet D, Chen HC, Tovchigrechko A, Vakser IA. DOCKGROUND resource for studying protein-protein interfaces. Bioinformatics 2006; 22:2612-2618.

[82] Breiman L. Bagging predictors. Mach Learn 1996;24:123-140. 
[83] Lee KH, Xie D, Freire E, Amzel LM. Estimation of changes in side chain configurational entropy in binding and folding: general methods and application to helix formation. Proteins 1994;20:68-84.

[84] Brady GP, Sharp KA. Entropy in protein folding and in protein-protein interactions. Curr Opin Struct Biol 1997;7:215-221.

[85] Doig AJ, Sternberg MJ. Side-chain conformational entropy in protein folding. Protein Sci 1995;4:2247-2251.

[86] Creamer TP. Side-chain conformational entropy in protein unfolded states. Proteins 2000;40:443-450.

[87] Kastritis PL, Moal IH, Hwang H, Weng Z, et al. A structure-based benchmark for protein-protein binding affinity. Protein Sci 2011;20:482-491.

[88] Schreiber G, Buckle AM, Fersht AR. Stability and function: two constraints in the evolution of barstar and other proteins. Structure 1994;2:945951.

[89] Moal IH, Bates PA. SwarmDock and the use of normal modes in proteinprotein docking. Int J Mol Sci 2010;11:3623-3648.

[90] Li X, Moal IH, Bates PA. Detection and refinement of encounter complexes for protein-protein docking: taking account of macromolecular crowding. Proteins 2010;78:3189-3196. 
[91] Torchala M, Moal IH, Chaleil RA, Fernandez-Recio J, Bates PA. SwarmDock: a server for flexible protein-protein docking. Bioinformatics 2013; 29:807-809.

[92] Hwang H, Vreven T, Janin J, Weng Z. Protein-protein docking benchmark version 4.0. Proteins 2010;78:3111-3114.

[93] Lensink MF, Mendez R, Wodak SJ. Docking and scoring protein complexes: CAPRI 3rd Edition. Proteins 2007;69:704-718.

[94] Dehouck Y, Grosfils A, Folch B, Gilis D, et al. Fast and accurate predictions of protein stability changes upon mutations using statistical potentials and neural networks: PoPMuSiC-2.0. Bioinformatics 2009;25:2537-2543.

[95] Janin J, Bahadur RP, Chakrabarti P. Protein-protein interaction and quaternary structure. Q Rev Biophys 2008;41:133-180.

[96] Jones S, Thornton JM. Principles of protein-protein interactions. Proc Natl Acad Sci USA 1996;93:13-20.

[97] Bickerton GR, Higueruelo AP, Blundell TL. Comprehensive, atomic-level characterization of structurally characterized protein-protein interactions: the PICCOLO database. BMC Bioinformatics 2011;12:313.

[98] Lo Conte L, Chothia C, Janin J. The atomic structure of protein-protein recognition sites. J Mol Biol 1999;285:2177-2198. 
[99] Andreani J, Faure G, Guerois R. Versatility and invariance in the evolution of homologous heteromeric interfaces. PLoS Comput Biol 2012; 8:e1002677.

[100] Ahmed MH, Spyrakis F, Cozzini P, Tripathi PK, et al. Bound water at protein-protein interfaces: partners, roles and hydrophobic bubbles as a conserved motif. PLoS ONE 2011;6:e24712.

[101] Langmuir I. The properties and structure of protein films. Proc Roy Inst Gt Britain 1938;30:483-496.

[102] Kauzmann W. Some factors in the interpretation of protein denaturation. Adv Protein Chem 1959;14:1-63.

[103] Lijnzaad P, Berendsen HJ, Argos P. Hydrophobic patches on the surfaces of protein structures. Proteins 1996;25:389-397.

[104] Lijnzaad P, Feenstra KA, Heringa J, Holstege FC. On defining the dynamics of hydrophobic patches on protein surfaces. Proteins 2008;72:105-114.

[105] Chandler D. Interfaces and the driving force of hydrophobic assembly. Nature 2005;437:640-647.

[106] Chan HS, Dill KA. Solvation: how to obtain microscopic energies from partitioning and solvation experiments. Annu Rev Biophys Biomol Struct 1997;26:425-459. 
[107] Janin J. A minimal model of protein-protein binding affinities. Protein Sci 2014;23:1813-1817.

[108] Finkelstein AV, Janin J. The price of lost freedom: entropy of bimolecular complex formation. Protein Eng 1989;3:1-3.

[109] Minh DD, Bui JM, Chang CE, Jain T, et al. The entropic cost of protein-protein association: a case study on acetylcholinesterase binding to fasciculin-2. Biophys J 2005;89:L25-27.

[110] Chang CE, McLaughlin WA, Baron R, Wang W, McCammon JA. Entropic contributions and the influence of the hydrophobic environment in promiscuous protein-protein association. Proc Natl Acad Sci USA 2008; 105:7456-7461.

[111] Cole C, Warwicker J. Side-chain conformational entropy at protein-protein interfaces. Protein Sci 2002;11:2860-2870.

[112] Grunberg R, Nilges M, Leckner J. Flexibility and conformational entropy in protein-protein binding. Structure 2006;14:683-693.

[113] Chothia C, Janin J. Principles of protein-protein recognition. Nature 1975; 256:705-708.

[114] Stites WE. Protein-Protein Interactions: Interface Structure, Binding Thermodynamics, and Mutational Analysis. Chem Rev 1997;97:1233-1250. 
[115] Schneidman-Duhovny D, Rossi A, Avila-Sakar A, Kim SJ, et al. A method for integrative structure determination of protein-protein complexes. Bioinformatics 2012;28:3282-3289.

[116] Moal IH, Jimenez-Garcia B, Fernandez-Recio J. CCharPPI web server: Computational Characterisation of Protein-Protein Interactions from structure. Bioinformatics 2014;:In press.

[117] Olechnovic K, Margelevicius M, Venclovas C. Voroprot: an interactive tool for the analysis and visualization of complex geometric features of protein structure. Bioinformatics 2011;27:723-724. 
Figure 1, An overview of the model derivation procedure, exemplified using the mutation of P1 leucine of Streptomyces griseus protease B (SGPB) to aspartic acid, in the interaction with the turkey ovomucoid third domain (OMTKY3). Voronoï diagrams (left) are constructed for the wild-type and mutant protein. The P1 residue is shown, as is OMTKY3 Pro-138, with which it interacts. The diagrams are used to calculate interaction type areas (centre), the difference between which are the changes in interaction type area upon mutation (top right). These are used to construct a linear equation for this mutant (bottom right). In this case, the mutation is dominated by a loss of $87.4 \AA^{2}$ area between neutral surfaces, and the gain of $84.7 \AA^{2}$ of interaction between neutral surface and H-bond acceptor surface, with smaller changes between other surfaces, such as acceptor-acceptor surface. Such an equation is generated for each mutation, which are collectively used to find the $\gamma$ values which best agree with the experimental $\Delta \Delta G$ data. Voronoï images created using Voroprot. 117

Figure 2: Surface energies values $\left(\hat{\gamma}, \mathrm{cal}^{\mathrm{m}} \mathrm{mol}^{-1} . \AA^{-2}\right)$ and standard deviations $(\sigma)$ derived from the bootstrap aggregating for all interaction types. Also shown are the sum of absolute changes in interaction area $\left(\mathrm{nm}^{2}\right)$.

Figure 3. Model applied to the absolute binding free energy data. (A) The validated set of high confidence affinities, with rigid complexes as black circles and the flexible as blue stars. Standard error in calculated $\Delta G_{\text {surf }}$ are estimated using $\sigma$ values derived from bootstrap aggregation, and error in experimental is set to 0.4 
kcal.mol ${ }^{-1}$ as suggested in Kastritis et al ${ }^{87}$ For the sake of clarity, error bars are omitted for the flexible complexes. (B) The model applied to the combined affinity benchmark, categorised as rigid high-confidence $\left(\mathrm{r}_{h c}\right)$, flexible high-confidence $\left(\mathrm{f}_{h c}\right)$, other rigid $\left(\mathrm{r}_{l c}\right)$, other flexible $\left(\mathrm{f}_{l c}\right)$, small (s) and large $(\mathrm{l})$.

Figure 4. Model applied to DockGround complexes, indicating the magnitude of the components in each of the 4434 interactions. Interactions are ordered by buried surface area. 


\begin{tabular}{c|ccccc} 
& $\mathrm{N}$ & $\mathrm{r}_{\text {Pred }}$ & $\mathrm{r}_{\mathrm{BSA}}$ & pPred & $\mathrm{p}_{\text {BSA }}$ \\
\hline $\mathrm{r}_{h c}$ & 23 & 0.71 & -0.70 & $<10^{-4}$ & 0.0001 \\
$\mathrm{f}_{h c}$ & 30 & 0.39 & -0.36 & 0.017 & 0.026 \\
$\mathrm{r}_{l c}$ & 42 & 0.48 & -0.47 & 0.0007 & 0.0009 \\
$\mathrm{f}_{l c}$ & 43 & 0.10 & 0.02 & 0.26 & 0.45 \\
$\mathrm{~s}$ & 72 & 0.37 & -0.41 & 0.0007 & 0.0002 \\
1 & 30 & 0.51 & -0.28 & 0.002 & 0.083 \\
$\mathrm{All}$ & 240 & 0.43 & -0.40 & $<10^{-4}$ & $<10^{-4}$
\end{tabular}

Table 1: Correlations and significance of correlations for the model applied to absolute binding free energy data, for the presented model and for buried surface area. Categories as per Figure 3 A.

\begin{tabular}{c|ccc|ccc} 
& \multicolumn{3}{c}{ Number } & \multicolumn{3}{c}{ Percent } \\
& High & Medium & Acceptable & High & Medium & Acceptable \\
\hline Top 1 & 0 & 7 & 14 & 0.0 & 5.7 & 11.4 \\
Top 5 & 3 & 20 & 33 & 2.4 & 16.3 & 26.8 \\
Top 10 & 5 & 28 & 46 & 4.1 & 22.8 & 37.4 \\
Top 50 & 5 & 46 & 77 & 4.1 & 37.4 & 62.6 \\
Top 100 & 5 & 57 & 101 & 4.1 & 46.3 & 82.1 \\
All & 7 & 71 & 121 & 5.7 & 57.7 & 98.4
\end{tabular}

Table 2: The performance of the model when applied to the ranking of docked poses. Success rates are shown in terms of the number of hits and the percentage of hits, in the top 1/5/10/50/100/all after clustering and ranking. Hits are defined as high quality, medium quality or better, and acceptable quality or better, as per the CAPRI criteria. 93 

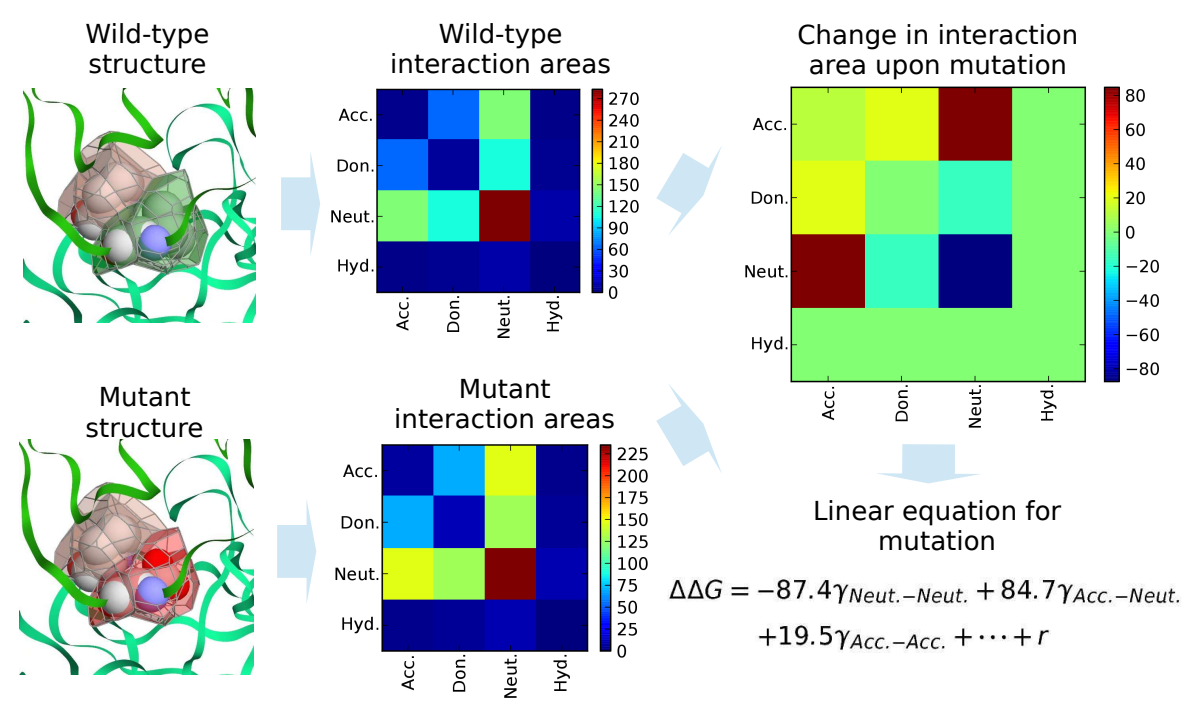

Figure 1: An overview of the model derivation procedure, exemplified using the mutation of P1 leucine of Streptomyces griseus protease B (SGPB) to aspartic acid, in the interaction with the turkey ovomucoid third domain (OMTKY3). Voronoï diagrams (left) are constructed for the wild-type and mutant protein. The $\mathrm{P} 1$ residue is shown, as is OMTKY3 Pro-138, with which it interacts. The diagrams are used to calculate interaction type areas (centre), the difference between which are the changes in interaction type area upon mutation (top right). These are used to construct a linear equation for this mutant (bottom right). In this case, the mutation is dominated by a loss of $87.4 \AA^{2}$ area between neutral surfaces, and the gain of $84.7 \AA^{2}$ of interaction between neutral surface and H-bond acceptor surface, with smaller changes between other surfaces, such as acceptor-acceptor surface. Such an equation is generated for each mutation, which are collectively used to find the $\gamma$ values which best agree with the experimental $\Delta \Delta G$ data. Voronoï images created using Voroprot.117 


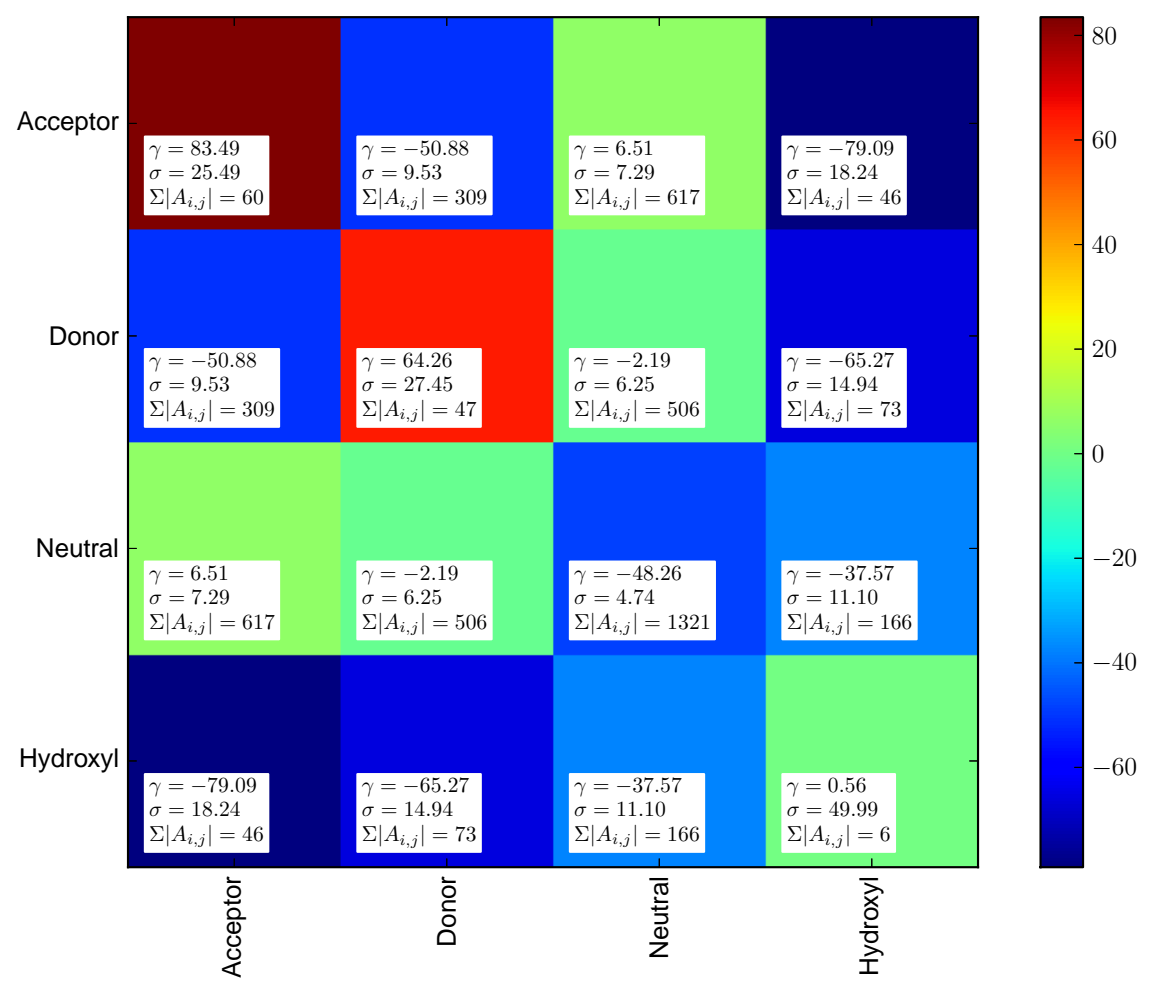

Figure 2: Surface energies values $\left(\hat{\gamma}\right.$, cal. $\left.m o l^{-1} . \AA^{-2}\right)$ and standard deviations $(\sigma)$ derived from the bootstrap aggregating for all interaction types. Also shown are the sum of absolute changes in interaction area $\left(\mathrm{nm}^{2}\right)$. 

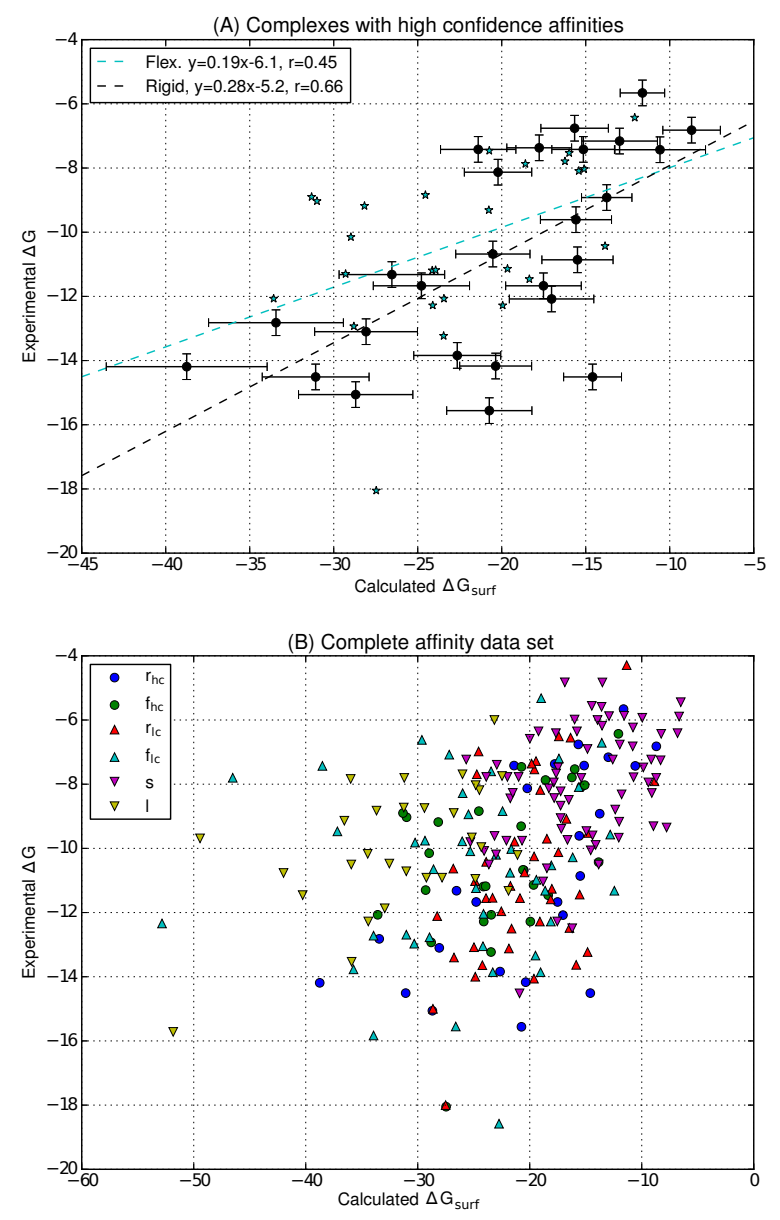

Figure 3: Model applied to the absolute binding free energy data. (A) The validated set of high confidence affinities, with rigid complexes as black circles and the flexible as blue stars. Standard error in calculated $\Delta G_{\text {surf }}$ are estimated using $\sigma$ values derived from bootstrap aggregation, and error in experimental is set to 0.4 kcal.mol ${ }^{-1}$ as suggested in Kastritis et al ${ }^{[87}$ For the sake of clarity, error bars are omitted for the flexible complexes. (B) The model applied to the combined affinity benchmark, categorised as rigid high-confidence $\left(\mathrm{r}_{h c}\right)$, flexible high-confidence $\left(\mathrm{f}_{h c}\right)$, other rigid $\left(\mathrm{r}_{l c}\right)$, other flexible $\left(\mathrm{f}_{l c}\right)$, small $(\mathrm{s})$ and large $(\mathrm{l})$. 


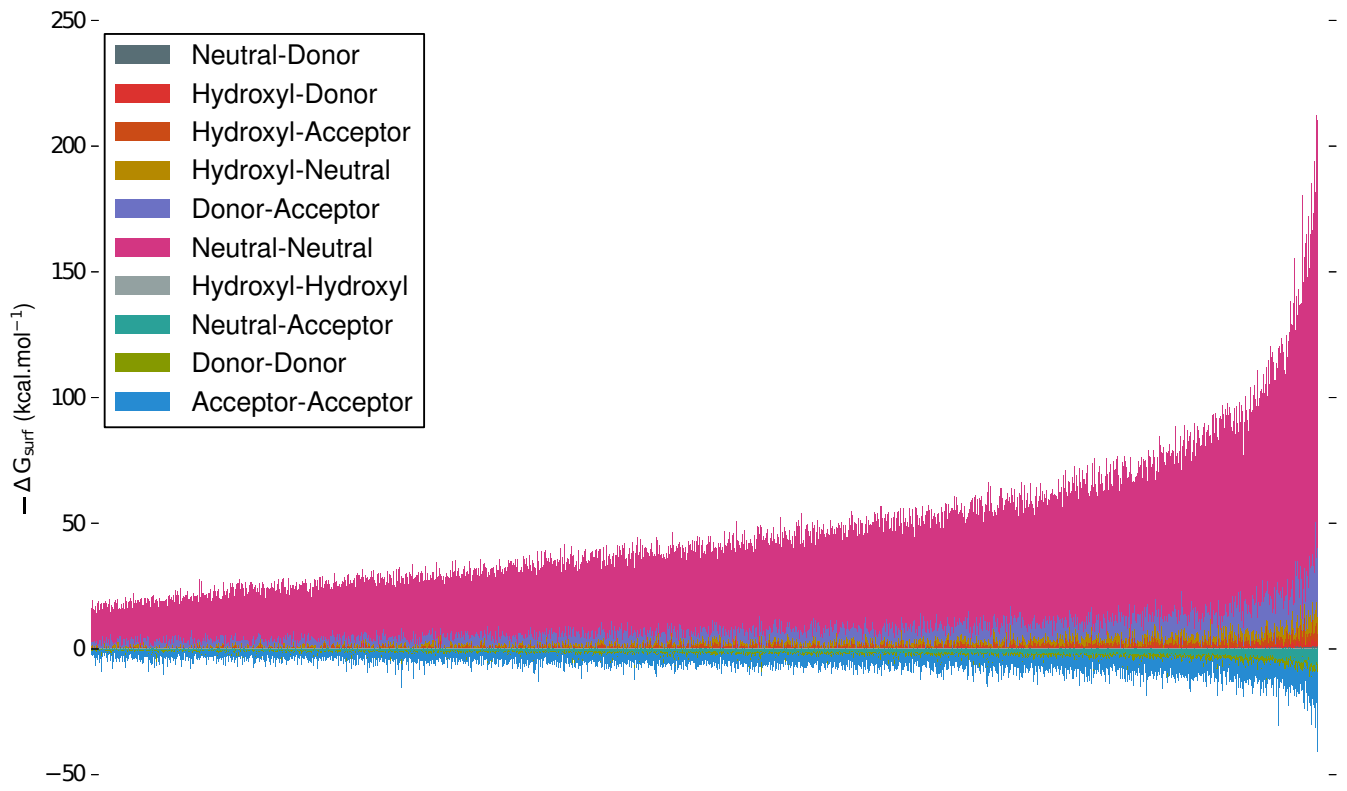

Figure 4: Model applied to DockGround complexes, indicating the magnitude of the components in each of the 4434 interactions. Interactions are ordered by buried surface area. 